\title{
Targeting of hepatic angiotensinogen using chemically modified siRNAs results in significant and sustained blood pressure lowering in a rat model of hypertension
}

\author{
Jeffrey Olearczyk ${ }^{1}$, Sheng Gao ${ }^{1}$, Marianne Eybye ${ }^{1}$, Satyasri Yendluri ${ }^{2}$, Lori Andrews ${ }^{2}$, Steven Bartz ${ }^{3}$, \\ Doris Cully ${ }^{1}$ and Marija Tadin-Strapps ${ }^{4}$
}

Angiotensinogen (AGT) is the precursor of active vasoconstrictive octapeptide angiotensin II (Ang II) in the renin-angiotensinaldosterone system. Blocking the AGT-converting enzymes in the pathway and the Ang II receptor through pharmacological agents has been proven to be effective in lowering blood pressure (BP) in hypertensive patients. In this study, we developed chemically modified small interfering RNAs (siRNA) to target hepatic AGT mRNA in rats. Lipid nanoparticle encapsulated siRNAs were efficiently delivered to rat liver and resulted in significant reduction in hepatic Agt mRNA levels and plasma AGT concentration without impairing liver function. Single intravenous injection of Agt siRNA led to significant and sustained BP lowering in spontaneous hypertensive rats and in Sprague-Dawley rats, and the effect was maintained by weekly siRNA dosing. Data presented here provide proof-of-feasibility for the use of siRNA technology for inhibition of peripheral AGT levels via hepatic mRNA silencing with beneficial effects on BP in preclinical rat models. Similar approach could be used for validation of novel hypertension hepatic and extrahepatic targets.

Hypertension Research (2014) 37, 405-412; doi:10.1038/hr.2013.155; published online 12 December 2013

Keywords: angiotensinogen; blood pressure lowering; lipid nanoparticles; siRNA targeting

\section{INTRODUCTON}

Hypertension is a chronic condition with a prevalence of $\sim 26 \%$ in the adult population worldwide. ${ }^{1}$ If untreated, hypertension can lead to death by stroke, heart or kidney failure. Currently, there are many antihypertension drugs on the market that primarily target the genes in the renin-angiotensin-aldosterone system (RAAS). RAAS is one of the important hormonal systems that regulates blood pressure (BP) as well as fluid and electrolyte homeostasis. ${ }^{2}$ Angiotensinogen (AGT) is a plasma glycoprotein primarily secreted by the liver and to a lesser extent by the kidney, heart, brain, adipose tissue and blood vessels. ${ }^{3}$ Through successive proteolytic action by renin and angiotensinconverting enzyme, AGT is processed into the active octapeptide angiotensin II (Ang II), which acts on Ang II receptor on various tissues and mediates vasoconstriction and aldosterone secretion, among many other functions. Agents against major RAAS pathway components have been successfully used for lowering BP and improving outcomes of cardiovascular disease (Figure 1). AGT, the precursor of active peptide and the most upstream component of RAAS pathway, has not been targeted so far, in part, due to the difficulty of modulating its expression through pharmacological interventions. ${ }^{4,5}$ Reduction in plasma AGT levels is expected to dampen the RAAS pathway activity, as other clinical agents do, and therefore lower BP. Preclinical studies have clearly linked the level of plasma AGT with BP regulation, namely: (1) Human plasma AGT concentration is close to the $\mathrm{K}_{\mathrm{m}}$ of renin, and therefore changes in the AGT level can lead to changes in the plasma Ang II level and subsequently $\mathrm{BP}^{6}{ }^{6}$ (2) Correlation of higher plasma AGT concentration and higher BP has been demonstrated in unrelated human subjects and in families; ${ }^{7-9}$ (3) Infusion of AGT in rats increases BP, whereas treatment with AGT antibody lowers BP; ${ }^{10,11}$ (4) Agt-knockout mice have reduced BP, whereas Agt overexpression by introducing up to four copies of $A g t$ gene in mice leads to a successive increase in BP with each additional copy of the gene. ${ }^{12-14}$ Therefore, modulation of AGT levels could be another promising approach to control BP. As AGT has proven to be difficult to target using conventional methodologies, $5,15,16$ its inhibition warrants the use of an alternative approach.

RNA interference (RNAi) is a post-transcriptional gene silencing mechanism that uses small double-stranded RNA molecules to direct gene silencing in a homology-based manner. Small interfering RNAs (siRNAs), recruit an RNA-induced silencing complex to the target mRNA, which then undergoes site-specific cleavage and degradation..$^{17,18}$

${ }^{1}$ Department of Hypertension, Merck Sharp \& Dohme Corp., Rahway, NJ, USA; ${ }^{2}$ Lead Development, Sirna Therapeutics Inc., a Wholly Owned Subsidiary of Merck Sharp \& Dohme Corp., San Francisco, CA, USA; ${ }^{3}$ Extarnal Scientific Affairs, Merck Sharp \& Dohme Corp., Palo Alto, CA, USA and ${ }^{4}$ Discovery Biology, RNA Therapeutics, Merck Sharp \& Dohme Corp., West Point, PA, USA

Correspondence: Dr M Tadin-Strapps, Discovery Biology, RNA Therapeutics, Merck Sharp \& Dohme Corp., 770 Sumneytown Pike PO BOX 4, West Point, PA 19486, USA. E-mail: marija_tadin-strapps@merck.com

Received 30 July 2013; revised 27 September 2013; accepted 23 October 2013; published online 12 December 2013 


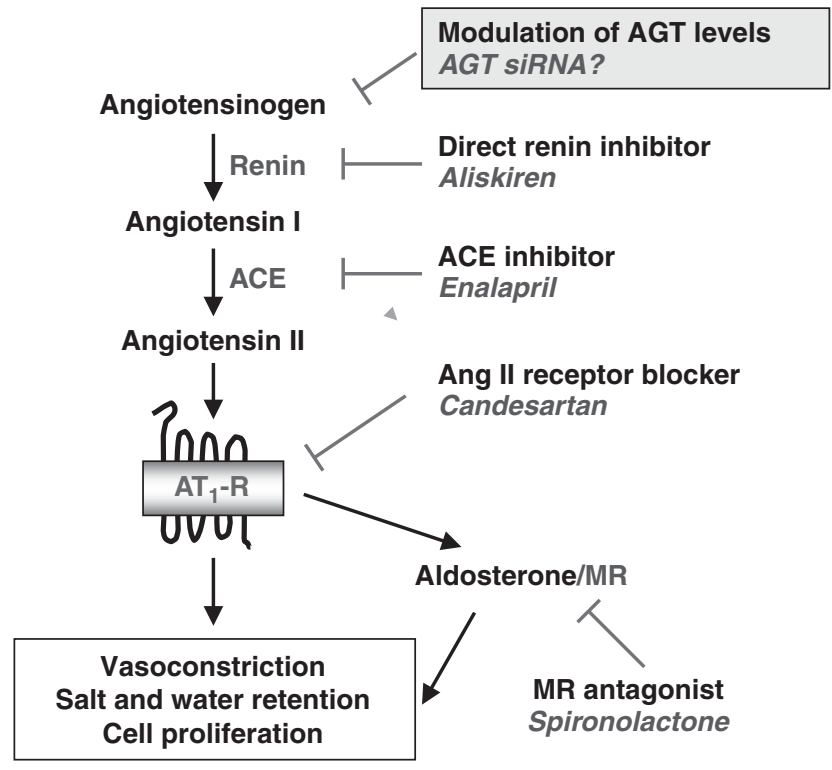

Figure 1 The RAAS pathway has long been targeted for antihypertensive therapies. Preclinical and clinical studies have demonstrated that inhibitors of the converting enzymes, renin and ACE, as well as the blockers of angiotensin II receptor and downstream mineralocorticoid receptor (MR) are very effective in lowering BP and improving cardiovascular outcomes. A full color version of this figure is available at the Hypertension Research journal online.

RNAi-mediated gene silencing has been widely used for both in vitro and in vivo target validation and mechanistic studies. For in vivo delivery, siRNAs are frequently packaged into cationic lipid nanoparticles, also known as lipid nanoparticles (LNPs). Systemic administration of LNPs has been successfully used for in vivo gene silencing in a number of preclinical species. ${ }^{19-25}$ siRNAs can be designed with high degree of specificity against target of interest, and new improvements in oligo design and chemical modifications have enabled the generation of oligos that are not immuno-stimulatory, show potent and durable target silencing and have minimal off-target effects. In addition, siRNAs can be designed against any complementary nucleotide sequence thus expanding the pool of potential drug candidates.

In this report, we demonstrate that chemically modified LNPencapsulated siRNAs can be successfully used for hepatic Agt targeting in rats with profound effects on circulating AGT levels and BP. The data demonstrate proof-of-feasibility for the use of siRNA technology for silencing of hepatic targets in the angiotensin-renin pathway as well as highlights the potential for AGT inhibition as means for treatment of hypertension.

\section{METHODS}

\section{siRNA characterization and synthesis}

All siRNAs used in this study were chemically synthesized and modified at Merck's oligosynthesis facility as previously described. ${ }^{21}$ The sequences of lead Agt siRNAs, non-targeting (nt) and positive controls used in this study are shown in Supplementary Table 1 (in the $5^{\prime}-3^{\prime}$ direction). siRNA sequences contained the following chemical modifications added to the $2^{\prime}$ position of the ribose sugar when indicated: deoxy (d), $2^{\prime}$ fluoro (flu) or $2^{\prime}$ O-methyl (ome). Modification abbreviations are given immediately preceding the base to which they were applied. Passenger strands are blocked with an inverted abasic nucleotide on the $5^{\prime}$ and $3^{\prime}$ ends (iB).

\section{Encapsulation of siRNA}

siRNAs used in in vivo studies were encapsulated into L201 lipid nanoparticle as previously described. ${ }^{26,27}$ L201 has the following chemical composition:
CLinDMA (2-\{4-[(3b)-cholest-5-en-3-yloxy]-butoxy\}-N,N-dimethyl-3-[(9Z,12 Z)-octadeca-9,12-dien-1-yloxy]propan-1-amine), cholesterol, and PEG-DMG (monomethoxy(polyethyleneglycol)-1,2-dimyristoylglycerol) in 50.3:44.3:5.4 molar ratio, respectively.

\section{Cell culture}

Rat hepatocyte cells were obtained from Cellz Direct and grown in Dulbecco's modified Eagle's medium (Mediatech, Manassas, VA, USA) supplemented with Cell Maintenance pack. Cells were plated $\left(2.4 \times 10^{4}\right.$ cells per well $)$ in BD Biosciences, Biocoat Collagen I-coated clear 96-well plates (BD Biosciences, San Jose, CA, USA). A total of 42 siRNA sequences were tested in an initial screening. Transfections were performed using Lipofectamine RNAiMAX Reagent as per the manufacturer's instructions (Life Technologies, Grand Island, NY, USA). Final siRNA concentration was $10 \mathrm{~nm}$ per well. All transfections were set up as biological replicas in two cell streams.

For concentration-response curves, cells were plated, cultured and transfected as described for the initial screening. Fourfold serial dilutions were tested for each siRNA with a starting concentration at $40 \mathrm{~nm}$ to generate a 12-point curve. Cell lysates were prepared using Biomek FX liquid handler and Cells-to-Ct Bulk Lysis Reagents (Life Technologies) according to the manufacturer's instructions. cDNA was generated using Ambion Cells-to-Ct Kit (Life Technologies). Taqman quantitative PCR analysis was done on ABI 7900 HT Real-Time PCR System. Reactions were set up in duplicate, singleplex with the final volume of $10 \mu \mathrm{l}$ using TaqMan Gene Expression Master Mix (Life Technologies). All Taqman probes and primers were purchased from Applied Biosystems as pre-validated sets: rat GAPDH Cat no. 4352338E; rat Agt Assay ID Rn00593114_m1 (Applied Biosystems, Foster City, CA, USA). Taqman data analysis was done using standard methods on an ABI 7900 Real-Time PCR System as described previously. ${ }^{28}$

\section{Experimental animals and animal care for phenotypic characterization}

Aged (1-1.5 years old) male Spontaneously Hypertensive rats (NTac:SHR, Taconic) weighing $380-420 \mathrm{~g}$ or male Sprague-Dawley rats (NTac:SD, Taconic) weighing $550-590 \mathrm{~g}$ were used. Animals were individually housed, allowed rodent chow (7012 Teklad LM-485 diet) and drinking water ad libitum and kept on a $12 \mathrm{~h}$ light:dark cycle. During study, active and non-targeting (nt) control LNPencapsulated siRNAs were administered via tail vein injections at $3 \mathrm{mg} \mathrm{kg}^{-1}$ for each dose. Blood and tissue samples were collected at specific time points. Hemodynamic parameters were monitored via telemetry device as described below. All animal procedures were approved by the Institutional Animal Care and Use Committee (IACUC) of Merck Sharp \& Dohme (West Point, PA, USA) and conducted in accordance with National Institutes of Health guidelines.

\section{Analysis of Agt mRNA knockdown in SHR rats}

Rats were dosed via tail vein injections with a single $3 \mathrm{mg} \mathrm{kg}^{-1}$ dose of LNPencapsulated siRNAs. Animals were sacrificed at specific time points following dosing. Liver samples were collected immediately following euthanasia. Total RNA was isolated from the liver tissue using the RNeasy 96 Tissue Kit (Qiagen, Valencia, CA, USA) according to the manufacturer's instructions. All RNA samples were treated with DNase I (Qiagen) on column for $15 \mathrm{~min}$ at room temperature. Final RNA eluted was quantified and normalized to a concentration of $50 \mathrm{ng}^{-1} \mathrm{l}^{-1}$. First strand cDNA was generated from $0.1 \mu \mathrm{g}$ of total RNA using Ambion Cells-to-Ct Kit (Life Technologies). Taqman quantitative PCR analysis was done on an ABI 7900 Real-Time PCR System. Reactions were set up in duplicate, singleplex with a final volume of $10 \mu \mathrm{l}$ using TaqMan Gene Expression Master Mix (Life Technologies). The Taqman probes used for the in vivo studies were the same as described for the in vitro screening.

\section{Chronic measurement of arterial pressure}

All surgical procedures were approved by the Merck Animal Use and Care Committee and in accordance to the IACUC guidelines. Animals were instrumented with PhysioTel PA-C40 transmitters from DSI (Data Sciences International, St Paul, MN, USA). The catheter from the telemetry device was placed into the abdominal aorta via the femoral artery. To access the femoral vessels, a $1.5-\mathrm{cm}$ incision was made across the left inner thigh. Using blunt 
dissection, a subcutaneous pocket was formed up toward the area between the caudal edge of the ribcage and the most cranial extension of the knee's range of motion. The transmitter body was inserted into the pocket and secured with silk sutures through the tissue surrounding the pocket entrance. Once the transmitter housing was in place, the femoral artery was gently separated from the vein and the catheter from the telemetry device was advanced through the femoral artery into the abdominal aorta just below the left kidney $(\sim 5.5-\mathrm{cm})$. To keep the catheter in proper orientation, the catheter stem was sutured to the surrounding muscle tissue and looped subcutaneously to prevent kinking. The wound was then closed with surgical staples. Animals were allowed to recover from the implantation surgery for 14 days before initiating studies. Animals were lightly anesthetized with isoflurane and dosed intravenously via tail vein injections with a single $3 \mathrm{mg} \mathrm{kg}^{-1}$ dose of LNP-encapsulated siRNA. Systolic, diastolic and pulse pressures as well as heart rate were monitored continuously from conscious, freely moving animals and recorded using Dataquest A.R.T software (version 4.1) (Data Sciences International).

\section{Measurement of circulating AGT}

Conscious animals were restrained and whole blood was collected via tail vein into EDTA coated BD Microtainer tubes (BD Biosciences). The plasma was separated from whole blood by centrifugation $\left(10 \mathrm{~min}, 4^{\circ} \mathrm{C}, 2000 \times \mathrm{g}\right)$. The plasma was aliquoted and stored at $-80^{\circ} \mathrm{C}$ until assayed. Quantitative determination of rat plasma AGT was performed using solid-phase sandwich enzyme-linked immunosorbent assay (Immuno-Biological Laboratories Co. Ltd., Minneapolis, MN, USA) according to the manufacturer's instructions.

\section{RESULTS}

Identification of Agt siRNA leads

A total of 42 siRNAs were designed to target rat Agt mRNA. Primary in vitro screening was performed in rat hepatocyte cells at $10 \mathrm{~nm}$ for each siRNA. Twelve siRNAs (29\%) showed over $80 \%$ target knockdown (KD) at $24 \mathrm{~h}$ post transfection (Figure 2a). Top four siRNAs (Seq 1-4) were then titrated in the same assay to confirm in vitro $\mathrm{KD}$ efficacy and to generate dose-response curve. All four siRNAs led to near complete $\mathrm{KD}$ at higher concentrations and calculated $\mathrm{IC}_{50}$ values were all in the sub-nanomolar range (Figure 2b).

These four siRNAs were then tested in spontaneous hypertensive rat (SHR) rats for in vivo KD efficacy. siRNAs were formulated into lipid nanoparticle 201 (LNP201). This LNP was shown previously to successfully deliver siRNAs to the liver and result in hepatic mRNA silencing. ${ }^{26-28}$ LNP201-siRNAs were injected intravenously into SHR rats at a single $3 \mathrm{mg} \mathrm{kg}^{-1}$ dose and hepatic mRNA expression levels examined on days 3, 7, 14 and 21 post dosing (Figure 2c).

All four siRNAs led to significant reductions in liver Agt mRNA levels with $>90 \%$ max silencing on day 3. Agt mRNA levels began to return to baseline on day 7 and approached pretreatment levels by day 21 . On the basis of in vitro and in vivo activity and potency data, Seq 2 was selected as the lead siRNA for further in vivo testing.

In vivo targeting of hepatic Agt mRNA results in significant reduction of plasma AGT levels without affecting the liver function Naive SHR animals were dosed with Agt Seq 2 LNP or non-targeting (nt) control LNP $\left(3 \mathrm{mg} \mathrm{kg}^{-1}, n=5-7\right.$ per group) and the plasma and liver were collected at $8 \mathrm{~h}, 24 \mathrm{~h}$ and 7 days post treatment to assess how changes in the liver Agt mRNA expression translate to AGT concentration in the plasma. Single intravenous injection of Agt LNP led to immediate and significant decrease in hepatic Agt mRNA levels
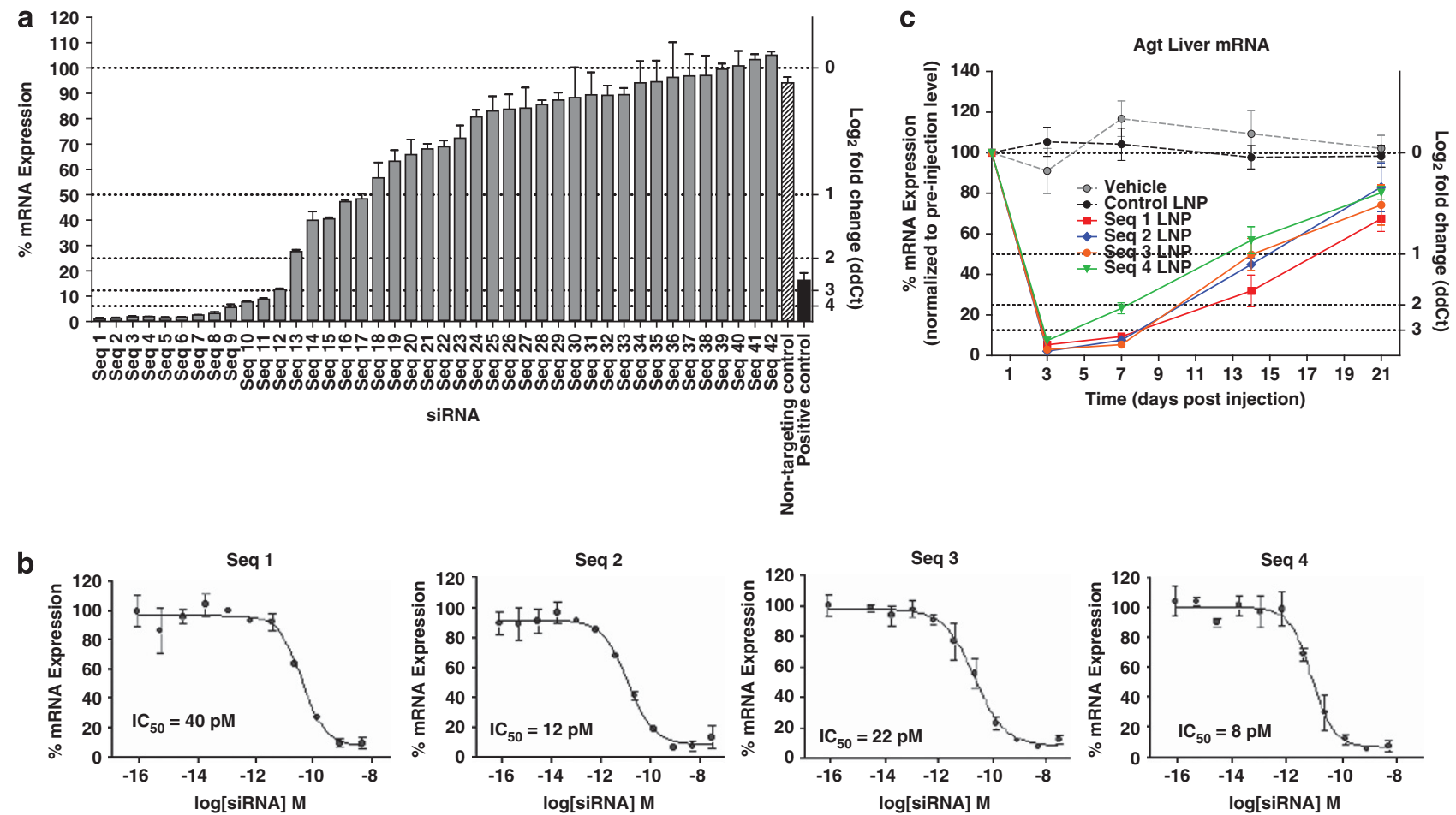

Figure 2 Selection of Agt siRNA. (a) Primary Agt siRNA screen in rat hepatocyte cells. Forty-two siRNAs designed against rat Agt were tested at 10 nm for Agt mRNA knockdown at $24 \mathrm{~h}$ post transfection. Average of two replicas \pm s.d. is shown. (b) Dose-response curves of top candidate siRNAs in rat hepatocyte cells. Top four Agt siRNAs were tested in fourfold serial dilution in order to determine $I_{50}$ values for each sequence. Calculated $I_{50 s}$ for all siRNAs were found to be in sub-nanomolar range. Average of two replicas \pm s.d. is shown. (c) In vivo efficacy of Agt siRNAs at day 3, 7, 14 and 21 post dosing in SHR rats. Rats were dosed intravenously (i.v.) with a single $3 \mathrm{mg} \mathrm{kg}^{-1}$ dose of Agt siRNA-LNPs, non-targeting control siRNA-LNP (nt control) or vehicle control. $N=3$ for each dose group at each time point. Group means \pm s.d. are shown. 
a

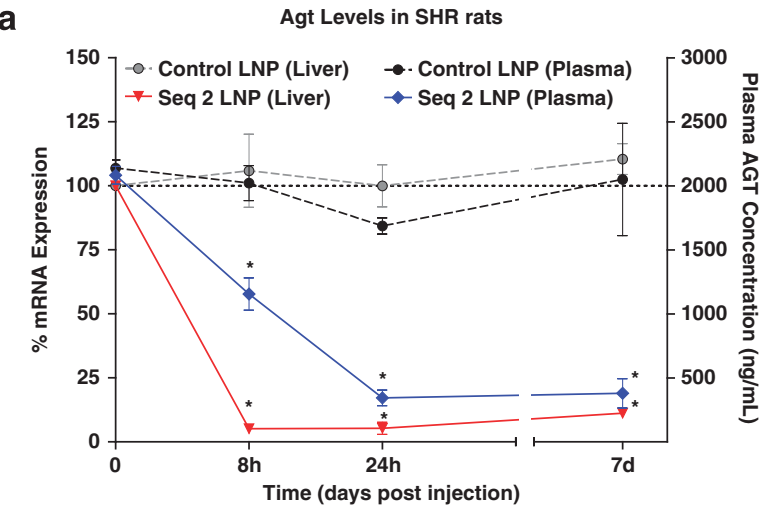

b

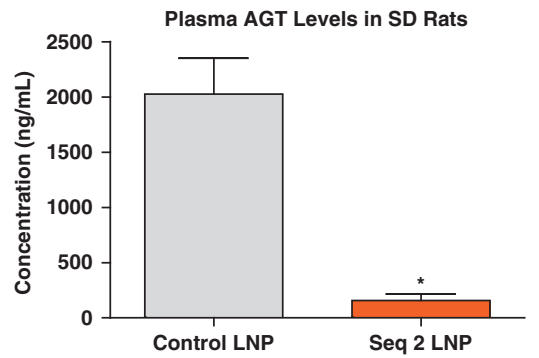

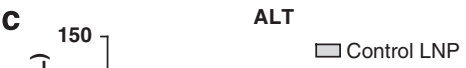

$\square$ Seq 2 LNP

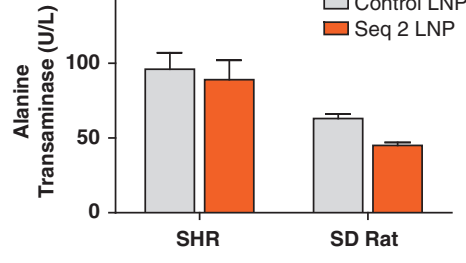

AST
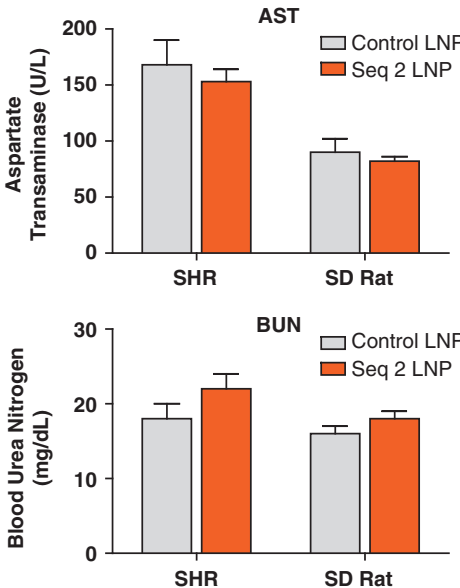

Figure 3 Agt siRNA-LNP dosing resulted in the lowering of plasma AGT levels in rats. (a) Reduction of hepatic Agt mRNA levels (left axis) and plasma AGT concentration (right axis) with Seq 2 siRNA LNP treatment in SHR. $N=5-7$ per group per time point. Mean \pm s.e.m. is shown. (b) Reduction of plasma AGT levels in SD rats 7 days after Seq 2 siRNA LNP administration. $N=5-8$ per group. Mean + s.e.m. is shown. (c) Alanine transaminase (ALT), aspartate transaminase (AST) and blood urea nitrogen (BUN) levels were not affected on day 7 after Agt siRNA administration in $S H R$ and $S D$ rats. ${ }^{*} P<0.001$ compared with control group, unpaired two-tailed $t$-test. Mean \pm s.e.m. is shown. Control LNP contains non-targeting (nt) control siRNA.

in SHR. Maximal reduction was achieved at $8 \mathrm{~h}$ post dose and was sustained for at least 7 days (Figure 3a). Agt Seq 2 group showed $\sim 95 \%$ target silencing for the first $24 \mathrm{~h}$ with $90 \%$ silencing remaining at day 7 post injection. No significant changes in hepatic Agt mRNA levels were observed in animals injected with nt control siRNA or vehicle (Figure 3a). Concentrations of circulating AGT were also significantly reduced by Agt siRNA injection. The level was 55, 17 and $18 \%$ of pretreatment level at $8 \mathrm{~h}, 24 \mathrm{~h}$ and 7 days post injection (Figure 3a). Compared with the kinetics of hepatic Agt mRNA silencing, the peak reduction of the plasma AGT levels occurred later at $24 \mathrm{~h}$ post dose, likely owing to time required for translation and secretion. Agt siRNA dosing resulted in similar reductions in the plasma AGT levels in SD rats 7 days after injection (Figure 3b) indicating that observed effects of hepatic Agt silencing are strainindependent. In addition, the plasma levels of alanine transaminase, aspartate transaminase and blood urea nitrogen were not significantly different between treatment groups in SHR and SD rats 7 days post treatment, suggesting that liver and renal functions were not altered with Agt siRNA treatment in both strains of rats (Figure 3c). Agt mRNA levels were not significantly altered in the adrenal gland, aorta, kidney cortex, kidney medulla, heart or fat with Agt LNP treatment (Supplementary Figure 1), which agrees with previous finding for LNP biodistribution showing liver and spleen as the main target organs. $^{29}$

In vivo targeting for hepatic Agt mRNA results in significant reduction of $\mathrm{BP}$ in hypertensive and normotensive rats

Treatment of SHR rats with Agt Seq 2 LNP $\left(3 \mathrm{mg} \mathrm{kg}^{-1}, n=5\right)$ significantly decreased systolic BP compared with control group (Figure 4a). The systolic BP reached nadir 4 days after treatment when compared with control-treated animals $(154 \pm 5 \mathrm{~mm} \mathrm{Hg}$ vs.
$188 \pm 1 \mathrm{~mm} \mathrm{Hg})$ and the decrease was sustained through day 7 after injection ( $152 \pm 4 \mathrm{~mm} \mathrm{Hg} v$ s. $188 \pm 2 \mathrm{~mm} \mathrm{Hg}$ ). Similar decreases were observed in mean and diastolic pressures (Figures $4 \mathrm{~b}$ and c). Three days after injection, there was a $10 \%$ increase in heart rate compared with control group ( $341 \pm 7$ b.p.m. vs. $308 \pm 8$ b.p.m.) that accompanied the decrease in BP and returned to pretreatment values by day 5 (Figure 4d).

To investigate whether the decrease in BP was unique to the SHR hypertensive model, BP was measured by telemetry in normotensive SD rats administered with Agt siRNA or nt control siRNA ( $3 \mathrm{mg} \mathrm{kg}^{-1}, n=7$ ). Similar to observations for changes in the plasma AGT levels in SHR and SD rats, Agt LNP treatment decreased systolic BP when compared with control animals (Figure 4e). Maximum systolic BP lowering was observed 4 days after treatment when compared with control group $(113 \pm 3 \mathrm{~mm} \mathrm{Hg}$ vs. $125 \pm 4 \mathrm{~mm} \mathrm{Hg})$ and was sustained for 7 days after treatment $(112 \pm 2 \mathrm{~mm} \mathrm{Hg} v s$. $126 \pm 3 \mathrm{mmHg}$ ). Similar decreases were observed in mean and diastolic pressures (Figures $4 \mathrm{f}$ and $\mathrm{g}$ ). Peak systolic BP lowering in SD rats was slightly lower than in SHR ( $14 \mathrm{~mm} \mathrm{Hg} v s .36 \mathrm{~mm} \mathrm{Hg}$ ). Changes in heart rate were not observed in Agt LNP-treated SD rats (Figure $4 \mathrm{~h}$ ), which could be attributed to the smaller magnitude of the BP decrease.

Reduction in BP following hepatic Agt targeting is sustained for 21 days and can be maintained by weekly repeat dosing

To understand the duration of BP lowering, a separate cohort of telemeterized SHR rats were treated with Agt or control LNP ( $3 \mathrm{mg} \mathrm{kg}^{-1}, n=7$ ) and BP monitored continuously. As in the previous study, systolic BP reached a nadir 4 days after treatment $(158 \pm 2 \mathrm{~mm} \mathrm{Hg}$ vs. $183 \pm 5 \mathrm{~mm} \mathrm{Hg})$ and was sustained through 7 days after injection (Figure 5a). Although systolic BP in the Agt LNP- 

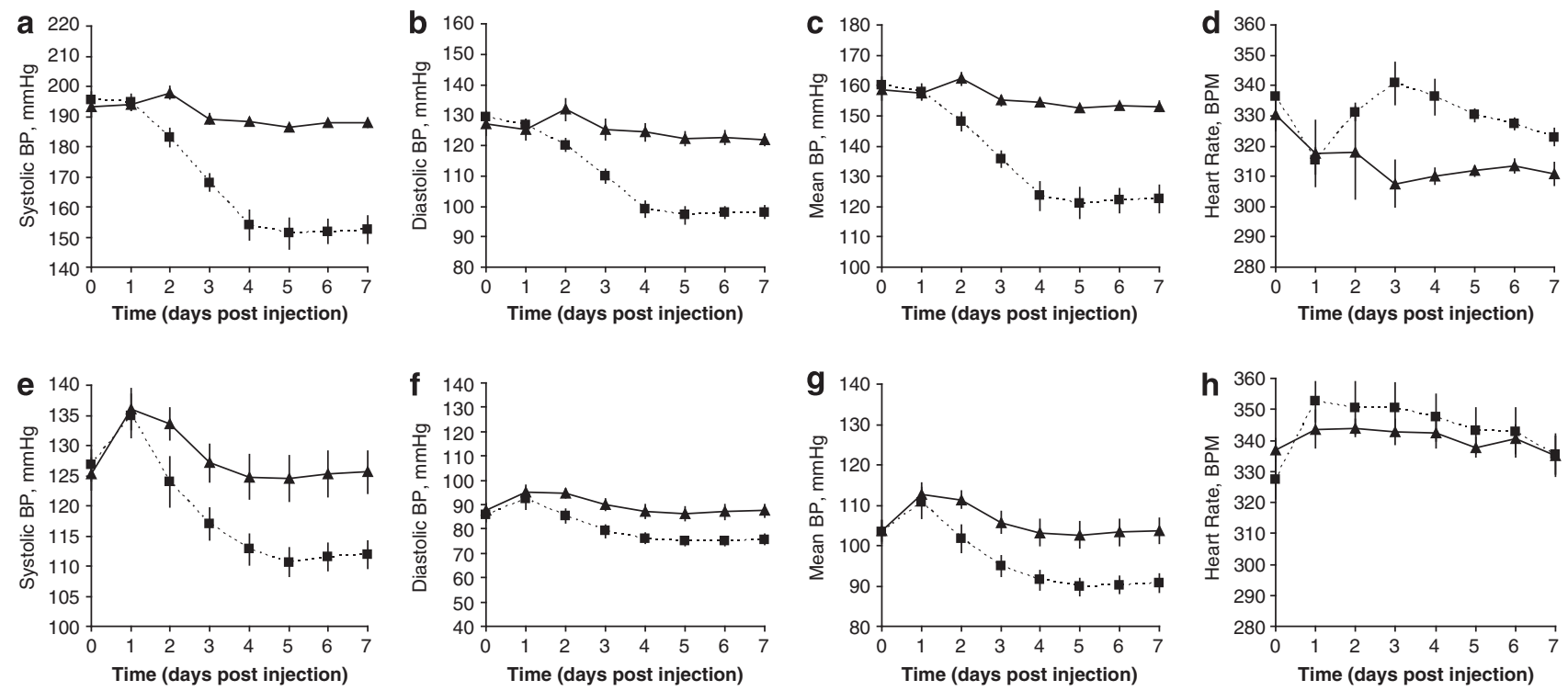

Figure 4 Agt siRNA-LNP dosing results in lowering of BP in rats. (a-d) Hemodynamic effects of Agt mRNA KD in aged SHR rats. $N=5$ per group. (e-h) Hemodynamic effects of Agt mRNA KD in SD rats. $N=8$ per group. $\mathbf{\Delta}$ : SHR or SD rats dosed with non-targeting (nt) control LNP; $\mathbf{\square}:$ SHR or SD rats dosed with LNP containing Agt Seq 2 siRNA.
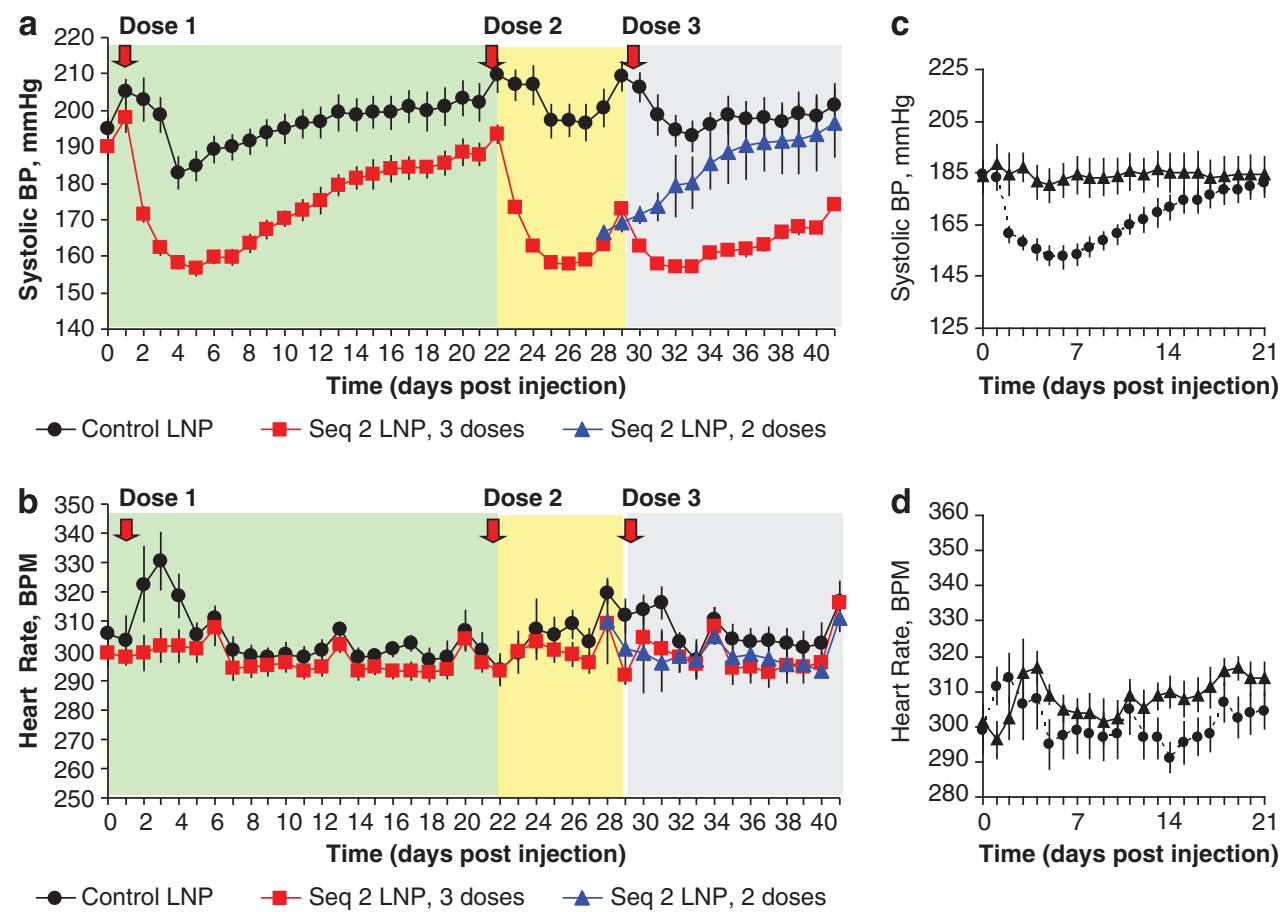

Figure 5 BP lowering can be maintained over time by repeated weekly Agt LNP dosing. Effects on BP are confirmed with a second independent siRNA lead. (a) Systolic BP and (b) HR of aged SHR rats treated with multiple doses of Agt Seq 2 LNP. $\bullet$ : Rats receiving three doses of non-targeting (nt) control LNP, $n=7$; $\mathbf{\square}$ : Rats receiving three doses of Agt Seq 2 LNP, $n=6$ until day 28, $n=4$ after day 28; $\mathbf{\Lambda}$ : Rats receiving first two doses of Agt Seq 2 LNP, $n=2$. (c) Systolic BP and (d) HR of aged SHR with single i.v. injection of Agt Seq 1 LNP at $3 \mathrm{mgkg}^{-1}$ dose. $N=8$ per group. $\boldsymbol{\Delta}$ : SHR dosed with non-targeting (nt) control LNP; $\mathbf{a}$ : SHR dosed with LNP containing Agt Seq 1 siRNA.

treated rats showed gradual increase back to baseline levels after day 7 , it was still lower than in control-treated animals 21 days post injection $(188 \pm 3 \mathrm{~mm} \mathrm{Hg}$ vs. $202 \pm 5 \mathrm{~mm} \mathrm{Hg})$. Similar changes were observed in mean and diastolic BPs (data not shown). A 10\% increase in heart rate was also observed on day 3 (331 \pm 10 b.p.m. vs. $302 \pm 6$ b.p.m.) that was consistent with the previous study and corresponded to the rapid decrease in BP at the same time (Figure 5b). The increase in heart rate returned to control values 5 days after treatment, also consistent with previous finding.

We next assessed weather BP lowering effect can be restored and maintained with repeat dose of Agt LNP. A second dose of Seq 2 or nt control LNP was administered to all animals at day 21, and then a 
third dose of LNPs was administered to a subset of animals on day 28. As observed with the initial dose, systolic BP reached a nadir again 4 days after the second dose and the third dose, to a comparable level as the first nadir (Figure 5a). No increase in heart rate was observed with the second and third doses, despite of significant BP lowering (Figure 5b). In rats that received second but not the third dose of Agt Seq 2 LNP, BP gradually increased over time, following similar kinetics as after the first dose. Repeated injection of nt control LNP had no significant effect on BP or heart rate.

To exclude the possibility that the observed pharmacological effects were specific to Agt Seq 2 oligo, the BP study was repeated in a separate cohort of telemetered SHR animals using a different LNPencapsulated Agt siRNA lead (Seq 1, $3 \mathrm{mg} \mathrm{kg}^{-1}$ intravenous injection). As shown in Figure 5c, Agt Seq 1 LNP decreased systolic BP similarly as was observed with Agt Seq 2 LNP. Systolic BP reduction reached its maximum 4 days after treatment and after 7 days gradually started coming back to pretreatment levels. Similar trends were also observed in mean and diastolic pressure (data not shown). These results confirmed that BP lowering observed with the lead Agt siRNA was due to reduction in Agt mRNA levels and not an off-target effect. Interestingly, changes in heart rate were not observed with Agt Seq 1 LNP treatment when compared with control animals, despite comparable BP decrease.

\section{BP lowering by hepatic Agt targeting can be further improved by candesartan}

Given the significant BP lowering effect in SHR by Agt siRNA alone, we set out to test whether this effect can be additive to that of currently used standard of care RAAS agent, such as candesartan. ${ }^{30-33}$ In SHRs that received Agt Seq 2 or nt control LNP on day 1, candesartan or placebo was administered orally at $3 \mathrm{mg} \mathrm{kg}^{-1}$ once daily from day 5 to 7 . Consistent with previous result, Seq 2 LNP led to about $50 \mathrm{~mm} \mathrm{Hg}$ maximal reduction in systolic BP and modest increase in heart rate. Addition of $3 \mathrm{mg} \mathrm{kg}^{-1}$ candesartan resulted in further BP lowering on top of Agt LNP, suggesting that the remaining RAAS pathway activity can be further blocked by candesartan (Figure 6a). Candesartan treatment also led to slight increase in heart rate, which could be attributed to baroreflex owing to additional BP lowering (Figure 6b).

\section{DISCUSSION}

Despite the fact that many antihypertensive therapeutic reagents are available today the health problems associated with hypertension continue to affect millions of people each year. Underlying causes for this gap include incomplete detection, poor compliance and lack of response to the current therapies. Thus, identification of new drugs with improved duration of effect and tolerability that may improve patient compliance is still of great interest. Recent development in RNAi technology provides unique benefits for new target identification and validation and the potential to use RNAi therapeutics for treatment of hypertension, because it allows for the development of highly selective reagents with potential of tissue-restricted targeting. ${ }^{34}$ Over the last decade, gene silencing methodologies have been used to silence target genes in the renin-angiotensin pathway in rat hypertension preclinical models. Antisense oligo deoxynucleotides, targeting AT1 receptors, AGT, angiotensin-converting enzyme and beta 1 -adrenergic receptors were shown to effectively reduce BP in rat hypertension models. ${ }^{15,35-40}$ Among these genes, AGT may be the best candidate for gene silencing approaches as the rest of these targets are also suitable for small-molecule inhibition, whereas inhibition of AGT via conventional approaches has proven unsuccessful thus far.
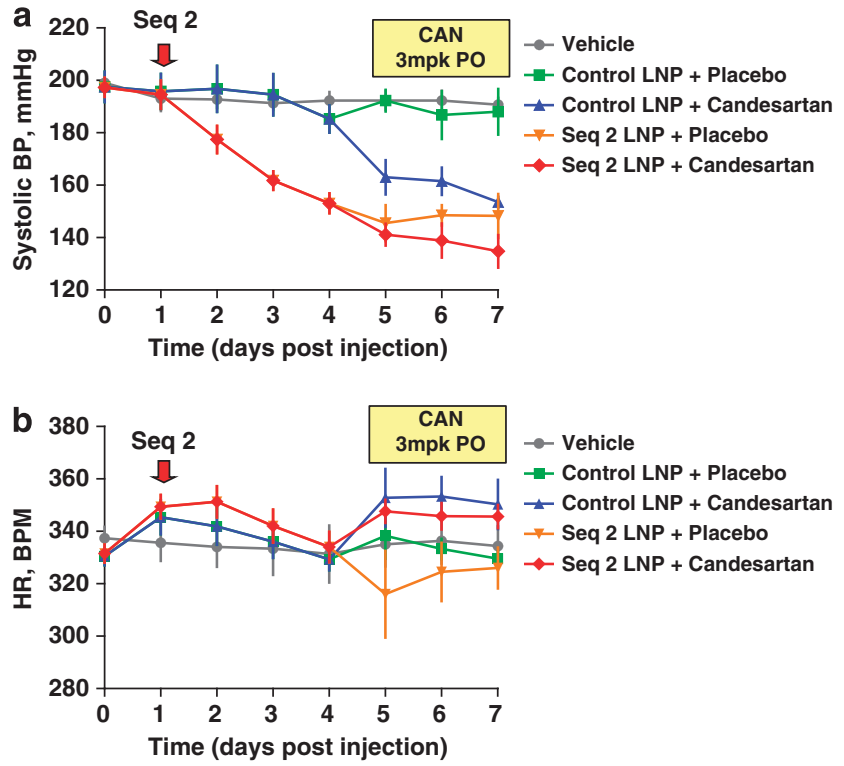

Figure 6 Candesartan further reduces BP in SHR rats treated with Agt Sec 2 LNP. (a) Systolic BP and (b) HR of aged SHR treated with $3 \mathrm{mg} \mathrm{kg}^{-1}$ Agt Seq 2 LNP followed by $3 \mathrm{mg} \mathrm{kg}^{-1}$ of candesartan. Vehicle group was injected with PBS vehicle via the tail vein on day 1 . The other groups were injected with $3 \mathrm{mg} \mathrm{kg}^{-1}$ non-targeting (nt) control or Agt Seq 2 LNP on day 1 and orally dosed with placebo or $3 \mathrm{mg} \mathrm{kg}^{-1}$ candesartan once daily on day 5,6 and $7 . N=4-6$ per group.

Here, we show for the first time that chemically modified LNPencapsulated siRNAs can be used to modulate AGT mRNA expression and plasma protein levels. We demonstrate potent and specific silencing of Agt mRNA in the liver with greater than 90\% maximal target knockdown. siRNA treatment was well tolerated and resulted in $>80 \%$ lowering of plasma AGT levels without affecting the liver and kidney functions. These effects were confirmed in both SHR and normotensive SD rats indicating that observed effects are not straindependent. Consistent with actions of other RAAS agents, effects on $\mathrm{BP}$ were more robust in SHR than in SD rats. Compared with earlier reports of Agt targeting in SHR rats using either LNP-antisense oligo deoxynucleotides or AAV-shRNA (adeno-associated virus-short hairpin RNA), we demonstrate superior BP-lowering efficacy $(\sim 50 \mathrm{~mm}$ $\mathrm{Hg} v$ s. $\sim 40$ and $\sim 30 \mathrm{~mm} \mathrm{Hg}$ respectively) and duration of effect following single dose (effects sustained for over 2 weeks $v$ s. few days). ${ }^{39,41,42}$

In order to confirm that the effects on BP and heart rate are Agtspecific, we repeated experiments with a second independent Agt siRNA (Seq 1). Both siRNAs showed very similar results with slight difference in maximal BP lowering $(\sim 40 \mathrm{~mm} \mathrm{Hg}$ for Seq 1 vs. $\sim 50 \mathrm{~mm} \mathrm{Hg}$ for Seq 2). BP effect of Seq 1 returned to baseline sooner than Seq 2 ( $\sim 15$ days for Seq 1$)$. These differences are likely due to variations in the potency and silencing kinetics that are inherent to each siRNA.

Interestingly, in SHR with Seq 2 siRNA treatment we observed transient but significant $\mathrm{HR}$ increase that coincided with the initial BP reduction and reached maximum just before reaching maximum BP effect. This HR increase was not observed in SD rats treated with Seq 2 siRNA or SHR treated with Seq 1 siRNA, or reported in published LNP-antisense oligo deoxynucleotides or AAV-shRNA experiments. It was evident that in the SHR treated with Seq 2 siRNA the extent and rate of BP lowering were greater than in other studies. It is possible that the fast and significant BP lowering evoked compensatory 
mechanism through baroreflex and that the HR effect became notable in the SHR study with Seq 2 siRNA due to its superior silencing potency and consequently greater BP effects.

Similar to earlier reports, we were able to maintain effects on BP with once weekly siRNA dosing. In our repeat dosing experiments, there appeared to be no desensitization to siRNA treatment as we were able to maintain BP lowering with multiple 1 per week dosing. No HR increase was observed after second and third doses, possibly due to lower predose BP and therefore small BP changes, or alternatively, baroreflex resetting after the first dose. Interestingly, maximal effect on BP lowering with multiple doses of siRNA was not improved beyond the level achieved following the first dose suggesting that maximal effect may have been achieved with this mechanism. In support of this notion is the fact that similar, albeit slightly less robust, maximal effect and duration of BP decrease were observed with other gene silencing approaches for either Agt or other targets in the RAAS pathway. ${ }^{37,43,44}$

In this report, we also compared the BP lowering efficacy of Agt siRNA therapy with the existing RAAS agent. Both Agt siRNA and $3 \mathrm{mg} \mathrm{kg}^{-1}$ of candesartan led to comparable max systolic BP lowering of $\sim 50 \mathrm{~mm} \mathrm{Hg}$ at day 7 (day 3 of candesartan dosing and day 6 post siRNA injection) (Figure 6a). Adding candesartan on top of Agt siRNA further lowered systolic BP. To look more closely at additivity of the two agents in this animal model additional dose titration and combination studies are required as the dose of candesartan used in this experiment is 'super-therapeutic'. In order to better understand the utility of Agt siRNA as a potential therapy, further studies are needed to determine the additivity of Agt siRNA on top of other standard of care agents, such as diuretics and calcium channel blockers. It will also be of value to monitor cardiac and renal functions in future studies with Agt siRNA, as prolonged hypertension are often associated with cardiac remodeling and renal impairment. ${ }^{45,46}$

We attempted to measure RAAS pathway parameters in some of the studies to further investigate the mechanism of action for Agt siRNA therapy. We observed a trend of reduction in plasma aldosterone levels 4 days after Agt siRNA-LNP injection (Supplementary Figure 2). The data were noisy mainly because of animal to animal variability and limitation of the enzyme immunoassay method. The lack of significant reduction in the plasma aldosterone level in spite of much lower angiotensinogen level could also reflect a compensatory mechanism on aldosterone synthesis and release, suggesting that concurrent therapy with mineralocorticoid receptor antagonist, ${ }^{47,48}$ such as spironolactone, may have a synergistic effect in BP lowering. We also attempted to measure plasma renin activity (PRA) in these studies. Our current PRA assay utilizes endogenous AGT, which is abundant under normal conditions, as substrate to measure enzyme activity of circulating active renin. However, when the plasma AGT levels are significantly ( $>90 \%$ ) reduced by siRNA knockdown, as was the case in the study samples, the assay could no longer accurately measure PRA likely due to shift in reaction equilibrium. In studies where SHRs were treated with ACEi (enalapril) ${ }^{49,50}$ and achieved comparable BP lowering (40-50 mm Hg), 10-fold increase in PRA was observed (data not shown). Similar increase in PRA would be expected in SHRs treated with Agt siRNA-LNP.

Current siRNA delivery methodologies have been most successful for the inhibition of liver targets. ${ }^{26,51}$ More recently some advancement has been made in extrahepatic delivery as well. ${ }^{52}$ We and others have recently showed that siRNA combinations are well tolerated in vivo in rodent models. ${ }^{53-55}$ One could envision using single-gene siRNA targeting and multiple-gene siRNA combinations in the AGT-renin pathway for target validation and mechanism of action studies both in the liver and extrahepatically. Such approaches are likely to accelerate identification of new single and combination drugs. In this report, we also show that siRNA administration can provide long-lasting BP lowering with a single injection and the effect can be sustained over extended period of time with repeated dosing. Although clinical safety and translatability remain to be determined, siRNA-LNP therapy would likely require much lower frequency of administration as compared with traditional RAAS blockers and therefore may increase patient compliance and improve BP control.

In summary, we show that targeting of Agt in the liver using LNPencapsulated chemically modified siRNAs can be used to achieve significant and sustained reduction in BP that is well tolerated, specific and reversible. Our findings highlight the potential for development of RNAi-based therapeutics for the treatment of hypertension as well as provide proof of concept for the use of siRNA technology for identification and validation of new hepatic hypertension targets.

\section{ACKNOWLEDGEMENTS}

We thank Sirna Formulation Chemistry for providing LNP formulations used in these experiments and Sirna In Vivo Biology for generation of in vivo samples for Agt siRNA evaluation in SHR rats. We also thank Laura SeppLorenzino for reviewing the manuscript and Walter Strapps for assistance with data analysis.

1 Kearney PM, Whelton M, Reynolds K, Muntner P, Whelton PK, He J. Global burden of hypertension: analysis of worldwide data. Lancet 2005; 365: 217-223.

2 Atlas SA. The renin-angiotensin aldosterone system: pathophysiological role and pharmacologic inhibition. J Manag Care Pharm 2007; 13(Suppl B), 9-20.

3 Corvol P, Soubrier F, Jeunemaitre X. Molecular genetics of the renin-angiotensinaldosterone system in human hypertension. Pathol Biol (Paris) 1997; 45: 229-239.

4 Brasier AR, Li J. Mechanisms for inducible control of angiotensinogen gene transcription. Hypertension 1996; 27(Pt 2), 465-475.

5 Morgan L, Broughton Pipkin F, Kalsheker N. Angiotensinogen: molecular biology, biochemistry and physiology. Int J Biochem Cell Biol 1996; 28: 1211-1222.

6 Gould $A B$, Green D. Kinetics of the human renin and human substrate reaction. Cardiovasc Res 1971; 5: 86-89.

7 Fasola AF, Martz BL, Helmer OM. Renin activity during supine exercise in normotensives and hypertensives. J Appl Physiol 1966; 21: 1709-1712.

8 Walker WG, Whelton PK, Saito H, Russell RP, Hermann J. Relation between blood pressure and renin, renin substrate, angiotensin II, aldosterone and urinary sodium and potassium in 574 ambulatory subjects. Hypertension 1979; 1: 287-291.

9 Watt GC, Harrap SB, Foy CJ, Holton DW, Edwards HV, Davidson HR, Connor JM, Lever AF, Fraser R. Abnormalities of glucocorticoid metabolism and the renin-angiotensin system: a four-corners approach to the identification of genetic determinants of blood pressure. J Hypertens 1992; 10: 473-482.

10 Gardes J, Bouhnik J, Clauser E, Corvol P, Menard J. Role of angiotensinogen in blood pressure homeostasis. Hypertension 1982; 4: 185-189.

11 Menard J, el Amrani Al, Savoie F, Bouhnik J. Angiotensinogen: an attractive and underrated participant in hypertension and inflammation. Hypertension 1991; 18: 705-707.

12 Kim HS, Krege JH, Kluckman KD, Hagaman JR, Hodgin JB, Best CF, Jennette JC, Coffman TM, Maeda N, Smithies O. Genetic control of blood pressure and the angiotensinogen locus. Proc Natl Acad Sci USA 1995; 92: 2735-2739.

13 Kimura S, Mullins JJ, Bunnemann B, Metzger R, Hilgenfeldt U, Zimmermann F, Jacob H, Fuxe K, Ganten D, Kaling M. High blood pressure in transgenic mice carrying the rat angiotensinogen gene. Embo J 1992; 11: 821-827.

14 Tanimoto K, Sugiyama F, Goto Y, Ishida J, Takimoto E, Yagami K, Fukamizu A, Murakami K. Angiotensinogen-deficient mice with hypotension. J Biol Chem 1994; 269: 31334-31337.

15 Makino N, Sugano M, Ohtsuka S, Sawada S. Intravenous injection with antisense oligodeoxynucleotides against angiotensinogen decreases blood pressure in spontaneously hypertensive rats. Hypertension 1998; 31: 1166-1170.

16 Phillips MI. Gene therapy for hypertension: sense and antisense strategies. Expert Opin Biol Ther 2001; 1: 655-662.

17 Aagaard L, Rossi JJ. RNAi therapeutics: principles, prospects and challenges. Adv Drug Deliv Rev 2007; 59: 75-86.

18 Tomari Y, Zamore PD. Perspective: machines for RNAi. Genes Dev. 2005; 19. $517-529$ 
19 Frank-Kamenetsky M, Grefhorst A, Anderson NN, Racie TS, Bramlage B, Akinc A Butler D, Charisse K, Dorkin R, Fan Y, Gamba-Vitalo C, Hadwiger P, Jayaraman M, John M, Jayaprakash KN, Maier M, Nechev L, Rajeev KG, Read T, Rohl I, Soutschek J, Tan P, Wong J, Wang G, Zimmermann T, de Fougerolles A, Vornlocher HP, Langer R Anderson DG, Manoharan M, Koteliansky V, Horton JD, Fitzgerald K. Therapeutic RNA targeting PCSK9 acutely lowers plasma cholesterol in rodents and LDL cholesterol in nonhuman primates. Proc Natl Acad Sci USA 2008; 105: 11915-11920.

20 Khoury M, Louis-Plence P, Escriou V, Noel D, Largeau C, Cantos C, Scherman D, Jorgensen C, Apparailly F. Efficient new cationic liposome formulation for systemic delivery of small interfering RNA silencing tumor necrosis factor alpha in experimental arthritis. Arthritis Rheum 2006; 54: 1867-1877

21 Morrissey DV, Blanchard K, Shaw L, Jensen K, Lockridge JA, Dickinson B, McSwiggen JA, Vargeese C, Bowman K, Shaffer CS, Polisky BA, Zinnen S. Activity of stabilized short interfering RNA in a mouse model of hepatitis B virus replication. Hepatology 2005; 41 . 1349-1356.

22 Sonoke S, Ueda T, Fujiwara K, Sato Y, Takagaki K, Hirabayashi K, Ohgi T, Yano J. Tumor regression in mice by delivery of $\mathrm{Bcl}-2$ small interfering RNA with pegylated cationic liposomes. Cancer Res 2008; 68: 8843-8851.

23 Zimmermann TS, Lee AC, Akinc A, Bramlage B, Bumcrot D, Fedoruk MN, Harborth J, Heyes JA, Jeffs LB, John M, Judge AD, Lam K, McClintock K, Nechev LV, Palmer LR, Racie T, Rohl I, Seiffert S, Shanmugam S, Sood V, Soutschek J, Toudjarska I, Wheat AJ, Yaworski E, Zedalis W, Koteliansky V, Manoharan M, Vornlocher HP, MacLachlan I. RNAi-mediated gene silencing in non-human primates. Nature 2006; 441: 111-114.

24 Arnold AS, Tang YL, Qian K, Shen L, Valencia V, Phillips MI, Zhang YC. Specific beta1adrenergic receptor silencing with small interfering RNA lowers high blood pressure and improves cardiac function in myocardial ischemia. J Hypertens 2007; 25 197-205

25 Morrissey DV, Lockridge JA, Shaw L, Blanchard K, Jensen K, Breen W, Hartsough K, Machemer L, Radka S, Jadhav V, Vaish N, Zinnen S, Vargeese C, Bowman K, Shaffer CS, Jeffs LB, Judge A, MacLachlan I, Polisky B. Potent and persistent in vivo anti-HBV activity of chemically modified siRNAs. Nat Biotechnol 2005; 23: 1002-1007.

26 Abrams MT, Koser ML, Seitzer J, Williams SC, DiPietro MA, Wang W, Shaw AW, Mao X, Jadhav V, Davide JP, Burke PA, Sachs AB, Stirdivant SM, Sepp-Lorenzino L. Evaluation of efficacy, biodistribution, and inflammation for a potent siRNA nanoparticle: effect of dexamethasone co-treatment. Mol Ther 2010; 18: 171-180.

27 Burchard J, Jackson AL, Malkov V, Needham RH, Tan Y, Bartz SR, Dai H, Sachs AB, Linsley PS. MicroRNA-like off-target transcript regulation by siRNAs is species specific. RNA 2009; 15: 308-315.

28 Tadin-Strapps M, Peterson LB, Cumiskey AM, Rosa RL, Mendoza VH, Castro-Perez J, Puig O, Zhang L, Strapps WR, Yendluri S, Andrews L, Pickering V, Rice J, Luo L, Chen Z, Tep S, Ason B, Somers EP, Sachs AB, Bartz SR, Tian J, Chin J, Hubbard BK, Wong KK Mitnaul LJ. siRNA-induced liver ApoB knockdown lowers serum LDL-cholesterol in a mouse model with human-like serum lipids. J Lipid Res 2011; 52: 1084-1097.

29 Shi B, Keough E, Matter A, Leander K, Young S, Carlini E, Sachs AB, Tao W, Abrams M, Howell B, Sepp-Lorenzino L. Biodistribution of small interfering RNA at the organ and cellular levels after lipid nanoparticle-mediated delivery. J Histochem Cytochem 2011; 59: 727-740

30 De Rosa ML. Cardio classics revisited-focus on the role of candesartan. Vasc Health Risk Manag 2010; 6: 1047-1063.

31 Fuchs B, Breithaupt-Grogler K, Belz GG, Roll S, Malerczyk C, Herrmann V, SpahnLangguth $\mathrm{H}$, Mutschler E. Comparative pharmacodynamics and pharmacokinetics of candesartan and losartan in man. J PharmPharmacol 2000; 52: 1075-1083.

32 Gleiter $\mathrm{CH}$, Morike KE. Clinical pharmacokinetics of candesartan. Clin Pharmacokinet 2002; 41: 7-17.

33 Khawaja Z, Wilcox CS. An overview of candesartan in clinical practice. Expert Rev Cardiovasc Ther 2011; 9: 975-982.

$34 \mathrm{Li} \mathrm{L}$, Shen Y. Overcoming obstacles to develop effective and safe siRNA therapeutics. Expert Opin Biol Ther 2009; 9: 609-619.

35 Gyurko R, Tran D, Phillips MI. Time course of inhibition of hypertension by antisense oligonucleotides targeted to AT1 angiotensin receptor mRNA in spontaneously hypertensive rats. Am J Hypertens 1997; 10(Pt 2), 56S-62S.

36 Wielbo D, Simon A, Phillips MI, Toffolo S. Inhibition of hypertension by peripheral administration of antisense oligodeoxynucleotides. Hypertension 1996; 28: 147-151.
37 Zhang YC, Bui JD, Shen L, Phillips MI. Antisense inhibition of beta(1)-adrenergic receptor $\mathrm{mRNA}$ in a single dose produces a profound and prolonged reduction in high blood pressure in spontaneously hypertensive rats. Circulation 2000; 101: 682-688.

38 He J, Bian Y, Gao F, Li M, Qiu L, Wu W, Zhou H, Liu G, Xiao C. RNA interference targeting the ACE gene reduced blood pressure and improved myocardial remodelling in SHRs. Clin Sci (Lond) 2009; 116: 249-255.

39 Tang X, Mohuczy D, Zhang YC, Kimura B, Galli SM, Phillips MI. Intravenous angiotensinogen antisense in AAV-based vector decreases hypertension. Am J Physiol 1999; 277 (Pt 2), H2392-H2399.

40 Xiao CS, Zhang JL, Qiu L. The effects of shRNA targeting angiotensin II type 1 receptor on blood pressure and AT1R mRNA expression in spontaneously hypertensive rats. Zhonghua Xin Xue Guan Bing Za Zhi 2007; 35: 354-358.

$41 \mathrm{Lu}$ P, Yuan L, Wang Y, Du Q, Sheng J. Effect of GPE-AGT nanoparticle shRNA transfection system mediated RNAi on early atherosclerotic lesion. Int J Clin Exp Pathol 2012; 5: 698-706.

42 Peng JF, Kimura B, Fregly MJ, Phillips MI. Reduction of cold-induced hypertension by antisense oligodeoxynucleotides to angiotensinogen mRNA and AT1-receptor mRNA in brain and blood. Hypertension 1998; 31: 1317-1323.

43 Makino N, Sugano M, Ohtsuka S, Sawada S, Hata T. Chronic antisense therapy for angiotensinogen on cardiac hypertrophy in spontaneously hypertensive rats. Cardiovasc Res 1999; 44: 543-548.

44 Zhou H, Bian YF, Li ML, Gao F, Xiao CS. Effects of RNA interference targeting angiotensin 1 receptor and angiotensin-converting enzyme on blood pressure and myocardial remodeling in spontaneous hypertensive rats. Zhonghua Xin Xue Guan Bing Za Zhi 2010; 38: 60-66.

45 Muiesan ML, Salvetti M, Rizzoni D, Paini A, Agabiti-Rosei C, Aggiusti C, Agabiti Rosei E. Resistant hypertension and target organ damage. Hypertens Res 2013; 36: 485-491.

46 Conrad CH, Brooks WW, Hayes JA, Sen S, Robinson KG, Bing OH. Myocardial fibrosis and stiffness with hypertrophy and heart failure in the spontaneously hypertensive rat. Circulation 1995; 91: 161-170.

47 Galuppo P, Bauersachs J. Mineralocorticoid receptor activation in myocardial infarction and failure: recent advances. Eur J Clin Invest 2012; 42: 1112-1120.

48 Zwadlo C, Bauersachs J. Mineralocorticoid receptor antagonists for therapy of coronary artery disease and related complications. Curr Opin Pharmacol 2013; 13: 280-286.

49 DiNicolantonio R, Doyle AE. Comparison of the actions of the angiotensin-converting enzyme inhibitors enalapril and S-9490-3 in sodium-deplete and sodium-replete spontaneously hypertensive rats. J Cardiovasc Pharmacol 1985; 7: 937-942.

50 Vincent ME, Swanson BN, Shepley K, Ferguson RK. Hemodynamic and humoral responses to enalapril and nifedipine in the rat. Clin Exp Hypertens A 1984; 6: 1485-1497.

51 Jayaraman M, Ansell SM, Mui BL, Tam YK, Chen J, Du X, Butler D, Eltepu L, Matsuda S, Narayanannair JK, Rajeev KG, Hafez IM, Akinc A, Maier MA, Tracy MA, Cullis PR, Madden TD, Manoharan M, Hope MJ. Maximizing the potency of siRNA lipid nanoparticles for hepatic gene silencing in vivo. Angew Chem Int Ed Eng/ 2012; 51 8529-8533.

52 Novobrantseva TI, Borodovsky A, Wong J, Klebanov B, Zafari M, Yucius K, Querbes W, Ge P, Ruda VM, Milstein S, Speciner L, Duncan R, Barros S, Basha G, Cullis P, Akinc A, Donahoe JS, Narayanannair Jayaprakash K, Jayaraman M, Bogorad RL, Love K, Whitehead K, Levins C, Manoharan M, Swirski FK, Weissleder R, Langer R, Anderson DG, de Fougerolles A, Nahrendorf M, Koteliansky V. Systemic RNAi-mediated gene silencing in nonhuman primate and rodent myeloid cells. Mol Ther Nucleic Acids 2013; 1: e4.

53 Ason B, Castro-Perez J, Tep S, Stefanni A, Tadin-Strapps M, Roddy T, Hankemeier T, Hubbard B, Sachs AB, Michael Flanagan W, Kuklin NA, Mitnaul LJ. ApoB siRNAinduced liver steatosis is resistant to clearance by the loss of fatty acid transport protein 5 (Fatp5). Lipids 2011; 46: 991-1003.

54 Tep S, Mihaila R, Freeman A, Pickering V, Huynh F, Tadin-Strapps M, Stracks A Hubbard B, Caldwell J, Flanagan WM, Kuklin NA, Ason B. Rescue of Mtp siRNAinduced hepatic steatosis by DGAT2 siRNA silencing. J Lipid Res 2012; 53: 859-867.

55 Zhou J, Neff CP, Liu X, Zhang J, Li H, Smith DD, Swiderski P, Aboellail T, Huang Y, Du Q, Liang Z, Peng L, Akkina R, Rossi JJ. Systemic administration of combinatorial dsiRNAs via nanoparticles efficiently suppresses HIV-1 infection in humanized mice. Mol Ther 2011; 19: 2228-2238.

Supplementary Information accompanies the paper on Hypertension Research website (http://www.nature.com/hr) 